\title{
BMJ Open Phase II clinical trial testing the safety of a humanised monoclonal antibody anti-CD20 in patients with heart failure with reduced ejection fraction, ICFEr-RITU2: study protocol
}

\author{
Luis Sánchez-Trujillo, Carlos Jerjes-Sanchez, ${ }^{\circledR}$ David Rodriguez, ${ }^{\circledR}$ \\ Jathniel Panneflek, ${ }^{\ominus}$ Claudia Ortiz-Ledesma, Gerardo Garcia-Rivas, ${ }^{\odot}$ \\ Guillermo Torre-Amione
}

To cite: Sánchez-Trujillo L, Jerjes-Sanchez C, Rodriguez D, et al. Phase II clinical trial testing the safety of a humanised monoclonal antibody anti-CD20 in patients with heart failure with reduced ejection fraction, ICFEr-RITU2: study protocol. BMJ Open 2019;9:e022826. doi:10.1136/ bmjopen-2018-022826

\section{- Prepublication history and} additional material for this paper are available online. To view these files, please visit the journal online (http://dx.doi org/10.1136/bmjopen-2018022826).

Received 9 March 2018 Revised 7 August 2018 Accepted 27 September 2018

Check for updates

(C) Author(s) (or their employer(s)) 2019. Re-use permitted under CC BY-NC. No commercial re-use. See rights and permissions. Published by BMJ.

Tecnologico de Monterrey, Escuela de Medicina y Ciencias de la Salud, Monterrey, N.L., Mexico

Correspondence to Dr Guillermo Torre-Amione; guillermo.torre@itesm.mx

\section{ABSTRACT}

Introduction Chronic heart failure with reduced ejection fraction (HFrEF) treatment targets neurohormonal inhibition; however, our experimental observations and the recent clinical evidence in myocardial infarction and heart transplant patients support the anti-inflammatory pathway as a potential novel therapeutic target. Therefore, we aimed to assess the safety of human monoclonal antibodyCD20 (rituximab) in patients with HFrEF.

Methods and analysis We designed this protocol according to the Standard Protocol Items: Recommendations for Interventional Trials guidelines as a phase II, single-centred, single group and prospective clinical trial. We hypothesise that the use of a monoclonal antibody, rituximab, could be a potentially safe new agent in HFrEF management. We will include patients with $\mathrm{EF} \leq 40 \%$, New York Heart Association functional class III/ IV and unresponsive to standard treatment. We will use a dosing regimen $(1000 \mathrm{mg}$ ) previously applied to posttransplant patients and patients with rheumatoid arthritis with favourable results, aiming to provide supplementary evidence of safety in patients with HFrEF. We designed strategies tailored to preserving the integrity of patient safety. The date of study initiation will be 29th of May 2019.

Ethics and dissemination The following protocol was approved by IRB committees, and as a requirement, all patients need to sign an informed consent form before being subjected to any procedure prior to the initiation of the study. We are aware that the trial will be run in patients who due to their cardiovascular functional class, have reserved prognosis, with no known therapy that leads to improvement. Hence, this trial searches to establish the safety of an alternative strategy in ameliorating prognosis. Regardless of the study outcomes, whether favourable or not, they will be published. If a favourable outcome is evidenced, it will prompt performing a phase III, efficacybased study.

Trial registration number The trial was approved by the IRB (CONBIOÉTICA-19-CEI-011-20161017 and COFEPRIS17-Cl-19-039-003), and registered at Clinicaltrials.gov (NCT03332888; Pre-Results).

\section{Strengths and limitations of this study}

- The study undertakes extensive safety measures to ensure participant safety.

- The use of biomarkers and imaging techniques such as MRI enable us to assess the impact the study drug could have on this group of patients, qualitatively and quantitatively.

- This endeavour is an investigator-initiated trial, with internal funding.

- The sample size is small, which would give us exploratory findings that could be used in larger trials.

- This is an open-label, unblinded trial with no control group.

\section{INTRODUCTION}

Currently, in chronic 'heart failure with reduced ejection fraction' (HFrEF), therapeutic management targets neurohormonal inhibition; however, the prevalence of postdischarge events, rehospitalisation and mortality rates remain high. ${ }^{1}$ New clinical and experimental evidence supports inflammation as the central mediator in the development and progression of HFrEF. ${ }^{2}$ Particularly, the B-cell activation signalling, which includes the production of pro-inflammatory cytokines, the propagation of anticardiac antibodies with the subsequent activation of the complement system. ${ }^{3}$ A large number of autoantibodies against specific cardiac antigens have been determined in patients with end-stage HFrEF. ${ }^{3}$ For instance, our group analysed human hearts with HFrEF and found that approximately $70 \%$ of them had anticardiac antibodies deposited within the myocardium. Additionally, the complement activity was also demonstrated in a third part of the biopsies. In fact, the presence of these molecules was directly correlated with the 
length, class and progressive state of the disease, indicating that these antibodies play an active role in the progression of HFrEF. ${ }^{14}$ Furthermore, in a murine model, we proved that by the depletion of B-lymphocytes using a monoclonal antibody that binds to the CD20 antigen (rituximab), we were able to reduce the expression of heart failure. We observed the cardioprotective effect of B-cell depletion by a significant reduction of cell hypertrophy, collagen deposition and by encountering a preserved ejection fraction in the CD20treated mice. ${ }^{25}$ Moreover, rituximab has been successfully used, in complicated postheart-transplant patients with severe heart failure due to transplant rejection, observing a regression of ventricular dysfunction in the majority. Similarly, rituximab has been efficient and safe in haematological malignancies and autoimmune diseases. ${ }^{6}$ Finally, the purpose of this trial is to test the hypothesis that the use of a monoclonal antibody, rituximab, is indeed safe in patients with HFrEF, as observed in myocardial infarction with high levels of high-sensitivity $\mathrm{C}$ reactive protein patients treated with a human monoclonal anti-interleukin (IL) $-\beta$ antibody. ${ }^{7}$ The trial is essential for further study, which would evaluate the efficacy of rituximab.

\section{STUDY DETAILS}

This study will be carried out in the Zambrano Hellion Hospital, under the supervision of Dr Guillermo TorreAmione. Additional information regarding healthcare resource utilisation is available in the subsection entitled 'Healthcare Resource Utilization' in the data repository in ClinicalTrials.gov. This study will be conducted per the Helsinki Declaration. Additional explanation and details are shown in the full version of this protocol in the online supplementary material.

\section{METHODS}

\section{Sample size}

We considered a convenience sample of 10 patients from our heart failure clinic since the previously reported incidence of acute coronary syndrome (ACS) and atrial and ventricular arrhythmias with the use of rituximab is $<0.1 \%$ and $7.6 \%$, respectively. ${ }^{8}$ Regarding the worsening of left ventricle ejection fraction (LVEF) attributed to rituximab, the incidence is limited to case reports only, wherefore it is impossible to precisely determine the incidence. ${ }^{9}$ Moreover, with the strategic safety measures that we will take before, during and following the rituximab administration, we aim to reduce the risk of potential cardiovascular adverse events (AEs). However, the sample size required; considering a power of $80 \%$ and an alpha score of 0.05 , obtained by probabilistic methods, ranges from 321 to 26091 subjects to enrol, which would, unfortunately, be out of our reach.

\section{Protocol information}

This protocol has been elaborated according to the Standard Protocol Items: Recommendations for Interventional
Trials guidelines drawn from the Equator Network (http://www.equator-network.org) and posteriorly registered at Clinicaltrials.gov on 2 November 2017, approved and given the identifier NCT03332888, Pre-results.

\section{Study design}

The study is a phase II, single-centred, single group, prospective clinical trial aiming to evaluate the safety of rituximab doses among stable patients with functional class III/IV according to the NYHA classification with HFrEF with an inadequate response to treatment. Inadequate response to treatment is being defined as hospitalisations and the worsening of functional class despite compliance and optimal dosage of standard treatment. Our protocol is a therapeutic exploratory phase II trial since our primary objective is to assess the safety of a predefined dose of rituximab in subjects with HFrEF. ${ }^{10}$ The index qualifying HFrEF must have $\mathrm{EF} \leq 40 \%$ based on echocardiographic or cardiac MRI techniques, aged $40-60$ years and being diagnosed $<12$ months before enrolment of study. ${ }^{11}$ Our selected inclusion, exclusion and elimination criteria can be seen in figure 1 . This study will not randomise the sample, wherefore allocation concealment is inapplicable. The reason, therefore, is due to the fragile nature of the study population, making it unethical to implement a control group. Investigators were not blinded due to the fact there is no group allocation during the study. This phase II trial aims to provide supplementary evidence of the safety of an already approved dosing regimen ${ }^{11}$ in a group of patients with severe HFrEF and unresponsive to standard treatment, with secondary end points of efficacy (analysed as exploratory). This trial is essential for the possible further analysis of the study drug for more extensive studies. The date of study initiation will be 29th of May 2019.

The primary objective of this clinical trial is to assess the safety, in the use of rituximab, in clinically stable patients, unresponsive to standard treatment and in functional class NYHA III-IV, under the standard of care therapy. As a secondary objective, we wish to explore the possibility of changes in the measurements of neurohormonal biomarkers considered to have a prognostic value in patients with HFrEF. Moreover, if the use of rituximab delays cardiac fibrotic remodelling, it will be evidenced on MRI.

\section{Patient and public involvement}

Patients were not involved in the idea conception, design or the protocol elaboration of this trial.

\section{Prescreening}

Prescreening is a key element to successfully identify the correct patients to be screened for this clinical study. We pay special attention, and apply scrutinous screening, to patients with recent history of a cardiovascular event, procedure or major surgical procedure in the last 30 days before screening. The prescreening visit will be used after review of the patient's charts to determine patient's 
Inclusion Criteria

1. Men and women (women not pregnant neither in lactation period) between $\geq 40$ and 60 years.

2. Diagnosis of HFrEF and NYHA functional class III-IV.

3 .EF $\leq 40 \%$ evaluated by MRI and or transthoracic echocardiography, with validity of 12 months.

4. Inadequate response to standard treatment.

5. Urgent medical attention or hospitalisation due to worsening of $\mathrm{HF}$ or $\mathrm{Ml}$ in the last 12 months.

6.Diagnosis of ischaemic or non-ischaemic dilated cardiomyopathy.

7.Life expectancy of at least 6 months, in investigator opinion.

8.Participants should sign an informed consent form (ICF) personally.

\section{Exclusion Criteria}

1.Severe primary valvular cardiopathy or valvular prosthesis (mechanical or bio-valve).

2. History of heart transplant surgery, cardiomyoplasty, left ventricular reduction surgery, valvuloplasty, implantation of a ventricle assist device and surgical cardiac congenital defect correction.

3.Any patient with recent or remote history of cardiac arrhythmias.

4. Implantable cardioverter defibrillator within the last 3 months.

5. Acute coronary syndromes that required pharmacological or mechanical reperfusion or medical treatment, within 30 days before selection.

6. Percutaneous coronary intervention within 30 days prior to selection.

7. Treatment with inotropic agents (dobutamine, milrinone, levosimendan), intravenous diuretics or vasodilators within 30 days of selection.

8.Pregnant women or breastfeeding period without adequate prenatal care, or of childbearing potential and are not practising an effective means of birth control.

9. Untreated thyroid disease.

10. Patients with glomerular filtration rate of $<30 \mathrm{~mL} / \mathrm{min}$, based on the Cockcroft-Gault formula

11. Rapidly progressive glomerulonephritis, seizures or psychosis, progressive neuropathy or myopathy.

12. Haemoglobin: $<85 \mathrm{~g} / \mathrm{L}$.

13. BNP elevation $>100 \mathrm{pg} / \mathrm{ml}$ or $>1.5$ its basal value.

14. WBC count $>5000 / \mathrm{mm}^{3}$.

15. Platelets: $<75000 / \mathrm{mm}$, AST or ALT >2.5 $\times$ upper limit of normal (ULN) unless related to primary disease.

16.Positive Hepatitis B or C serology (Hep B Surface antigen and Hep $\mathrm{C}$ antibody).

17. History of positive HIV.

18. Treatment with any investigational agent within 4 weeks of screening or 5 half-lives of the investigational drug (whichever is longer)
19. Recipients of an available attenuated vaccine within 4 weeks prior to randomisation.

20.Previous treatment with HMA-CD20 or any immunotherapy.

21. History of severe allergic or anaphylactic reactions to humanised or murine monoclonal antibodies.

22. Known active bacterial, viral, fungal (excluding fungal infections of nail beds/onychomycosis), mycobacterial, or other infection (including tuberculosis or atypical mycobacterial disease) or any major episode of infection requiring hospitalization or treatment with intravenous antibiotics within 4 weeks of screening, or oral antibiotics within 2 weeks before screening.

23. Consistent steroid administration within the past 4 weeks.

24. Lack of peripheral venous access.

25. Concomitant or previous malignancies, except curatively resected non-melanoma skin carcinomas or carcinoma in situ of the cervix.

26. History of psychiatric disorder that would interfere with participation in this protocol, such as depression, bipolar syndrome, schizophrenia.

27. Patients with concomitant severe COPD or emphysema.

28. Any other disease, metabolic dysfunction, physical examination finding or clinical laboratory finding giving reasonable suspicion of a disease or condition that contraindicates the use of an investigational drug or that may affect the interpretation of the results or render the patient at high risk from treatment complications.

29. Fertile female participants not using an approved anticonceptive method described in screening section.

30. Inability to comply/assist with study and follow-up procedures.

31. Any person who is not able to give adequate ICF.

Figure 1 Inclusion, exclusion, and elimination criteria. ALT, alanine transaminase; AST, aspartate transaminase; BNP, B-type natriuretic peptide; COPD, chronic obstructive pulmonary disease; HF, heart failure; HFrEF, heart failure with reduced ejection fraction; MI, myocardial infarction; MRSA, Methicillin-resistant Staphylococcus aureus; NYHA, New York Heart Association; VRSA, vancomycin-resistant $S$. aureus; WBC, white blood cell. 
eligibility and to obtain a baseline chest X-ray, Quantiferon, 24-hour Holter and baseline ECG, which is to be run locally, for those potentially eligible patients who do not have a current one available. These procedures will be carried out 14 days before the screening phase.

\section{Screening}

Screening evaluations will include extensive medical history assessment while paying particular attention to infectious history, both hepatic and kidney function, as well as possible oncologic disease. The time between screening and initiation can be up to approximately 2 weeks. Patients will be numbered and added to the database as soon as eligibility assessments have been completed. Rescreening is not allowed. Patients should be clinically stable at the time of screening.

Patients will be interrogated to rule out history of irregular heartbeats or chest pain of recent onset, lung or kidney disease, a weakened immune system and history of any of the following severe infections including: a) hepatitis B virus; b) hepatitis $\mathrm{C}$ virus; c) cytomegalovirus; d) herpes simplex virus; e) parvovirus $\mathrm{B} 19$; f) varicella-zoster virus (chickenpox or shingles); g) West Nile virus. Also, history of a recent vaccination or someone in the household who are scheduled to receive vaccinations. This is important since some types of vaccines can lead to the spread of diseases to individuals with weakened immune systems. Fertile women should use dual birth control (ie, condom plus diaphragm) while using rituximab and for 12 months after finishing treatment.

\section{Patient enrolment}

Enrolment will include a 7-day interval between the first five patients. Thereafter, an interim analysis will be conducted to uncover AEs and negative outcome attributable to the study drug, which could avoid unnecessary patient exposure to a hidden hazard. If the interim analysis evaluations results are favourable, the enrolment will proceed until the sample size is complete which can be seen in figure 2.

\section{Interim analyses}

An interim analysis of safety and futility will be carried out during the study to avoid exposing study patients to potentially dangerous treatment or unnecessary risks. We will perform an interim analysis when five of the subjects enrolled in the study hit the 3-month mark after initiation of the study. The aim is to examine the effects of rituximab treatment on key safety end points; incidence of potentially fatal ACS and arrhythmias as cardiovascular events. If more than three patients present one of these potentially fatal AEs, the intervention will be stopped to avoid compromising the safety of the patient. Despite discontinuing the intervention, patients enrolled in the study will continue with follow-up visits indefinitely at the Zambrano Hellion Hospital.

\section{Patients who discontinue study treatment}

It is important to note that participants may always withdraw consent or opt to retire from this trial at any given point in time. This will not impact their relationship with their physician; neither will it impact the quality and flow of their healthcare. Any patient who stops study treatment or withdraws consent will not be allowed to restart the study treatment. Patients who decide to discontinue study treatment, whatever the cause, will be granted scheduled follow-up visits, and be eligible to be admitted to the emergency department of the Zambrano Hellion Hospital under any protocol-related circumstance that may be presented. Regarding data of patients who decided to withdraw the protocol, the analysis of data will be performed using the on-treatment method.

\section{TREATMENT AND INTERVENTION PROTOCOL Study intervention}

In the papers published by Sánchez-Trujillo et al, Kaya $e t a l$ and Ludwig $e t a l$, we encountered the relationship that myocardial fibrosis had with B-type lymphocyte antibodies. ${ }^{2-4}$ These findings included the ways B-type lymphocyte antibodies could be involved in mediating the inflammatory pathway. Rituximab is one of the four monoclonal antibodies, which leads to the depletion of solely the B-type lymphocytes. However, ibritumomab, for instance, is given only as part of a treatment regimen in combination with the drug rituximab, in the treatment of certain types of non-Hodgkin's lymphoma. ${ }^{12}$ Another monoclonal antibody, I-131 tositumomab, is used in patients with CD20-positive follicular non-Hodgkin's lymphoma refractory to rituximab, ${ }^{13}$ and finally, alemtuzumab, additionally targets CD52 and is used in multiple sclerosis. ${ }^{14}$ We conclude that monoclonal antibodies cannot necessarily be compared since they have different therapeutic objectives and pathways. Since rituximab matched our aims and objectives, it was our study drug of choice.

We will enrol patients unresponsive to standard treatment and hospitalised in the last 12 months. Study

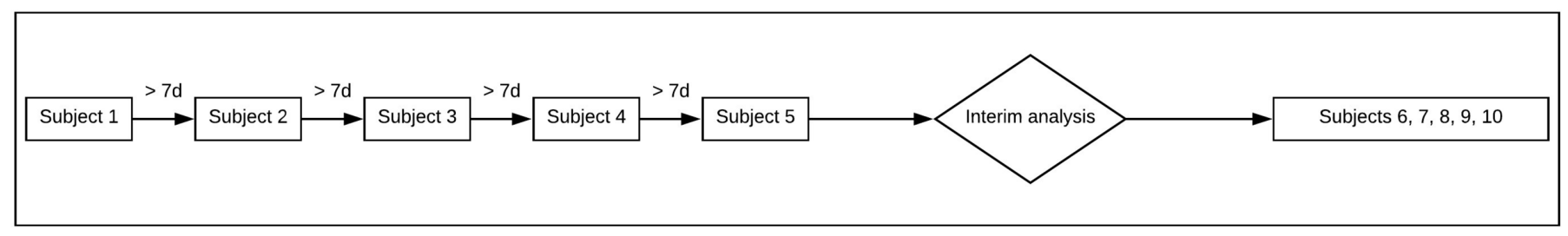

Figure 2 Enrolment plan. 

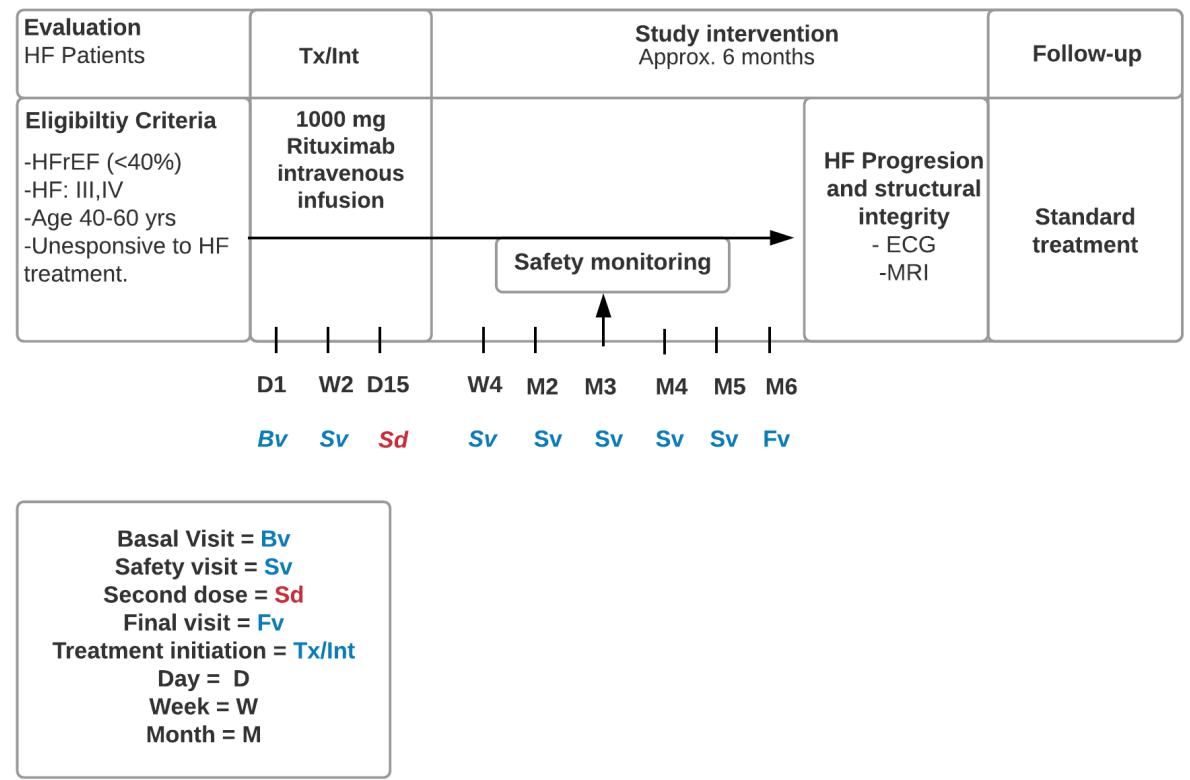

Figure 3 Study outline flow chart. HF, heart failure; HFrEF, heart failure with reduced ejection fraction.

treatment will be administered at the clinic under the supervision of the investigative staff with the dosing record captured within each visit on the study database. The study consists of two infusions, with study drug being administered on days 1 and 15 , respectively. After that, participants will be followed for 6 months where safety will be evaluated, to assess if any end points had been met. Patients will have scheduled assessments weekly after the initiation of treatment and monthly visits thereafter to evaluate the safety and non-occurrence of any mentioned plausible AEs which could be identified as trial end points. Despite discontinuing the intervention, patients enrolled in the study will continue with follow-up visits indefinitely at the Zambrano Hellion Hospital. The design of the study including screening phase, drug administration date and safety monitoring is presented in figure 3 .

\section{Dosage justification}

In the randomised, double-blind study to evaluate the effect of various re-treatment regimens of MabThera in combination with methotrexate on treatment response in rheumatoid arthritis patients with an inadequate response to methotrexate (MIRROR study), the higher rituximab dose group (two doses of $1000 \mathrm{mg}$ ) was associated with a safe and sustained clinical response at week 48 and was related with the lowest incidence of cardiovascular AEs. ${ }^{11}$ Aside from the fact that these patients submitted to this regimen had no significant elevation in their incidence of cardiovascular AEs, we prioritised the potential benefits that rituximab could have for these patients with class III and IV heart failure. Moreover, intravenous methylprednisolone $(100 \mathrm{mg})$ as premedication to rituximab infusion will be given to reduce the possibility and severity of infusion reactions. Acetaminophen and antihistamines have also been routinely given in clinical trials before rituximab infusions and are therefore also acceptable. ${ }^{15}$ Clinical staff will be entrusted to the study drug administration, patient monitoring and $\mathrm{AE}$ management and reporting. The detailed schedule of enrolment, interventions and visits for study participants is presented in figure 4 .

\section{Preparation}

The appropriate aseptic technique will be used while preparing rituximab for administration. To prepare the study drug, we will proceed according to the detailed preparation procedures described previously by Specks $e t$ $a l^{16}$ We will withdraw the required amount of rituximab, diluting it to its final concentration of $1-4 \mathrm{mg} / \mathrm{mL}$ into an infusion bag with $0.9 \%$ sodium chloride, USP. After carefully mixing the solution it will be ready for use. Unused remnants left in the phial will be discarded. ${ }^{17}$ We will inspect for discolouration or particulate matter, and protect vials from direct sunlight, storing it at its stable temperature at $2^{\circ} \mathrm{C}-8^{\circ} \mathrm{C}\left(36^{\circ} \mathrm{F}-46^{\circ} \mathrm{F}\right)$. Due to rituximab having such a delicate nature, it will be refrigerated inside a pharmacy granted to comply with all of these instructions. ${ }^{1516}$ The diluted solution will be stored in the same location following storage instructions. The vials provided to the pharmacy will have study-specific investigational agent labels, with corresponding national registration number 248M98 SSA.

\section{Infusion monitoring and application}

After preparation of the study drug, the infusion will take place in a controlled and specialised environment in the Zambrano Hellion Hospital, where investigators staff is equipped and prepared to infuse and treat patients in the 


\begin{tabular}{|c|c|c|c|c|c|c|c|c|c|c|c|c|c|c|}
\hline \multirow[b]{2}{*}{ Visits } & \multirow[t]{2}{*}{$\begin{array}{l}\text { Pre- } \\
\text { screening }\end{array}$} & \multicolumn{2}{|c|}{$\begin{array}{l}\text { Screening and } \\
\text { Selection }\end{array}$} & \multirow{2}{*}{\begin{tabular}{c|} 
Proc 1 \\
$\sqrt{3}$
\end{tabular}} & \multirow{2}{*}{$\frac{\text { Sv1 }}{\text { v4 }}$} & \multirow{2}{*}{$\frac{\text { Proc } 2}{\mathrm{v} 5}$} & \multirow{2}{*}{$\frac{\mathrm{sv2}}{\mathrm{v} 6}$} & \multirow{2}{*}{$\frac{s \sqrt{3}}{\sqrt{7}}$} & \multirow{2}{*}{$\frac{\text { Sv4 }}{\text { v8 }}$} & \multirow{2}{*}{$\frac{\text { Sv5 }}{\text { v9 }}$} & \multirow{2}{*}{$\frac{\text { Sv6 }}{\text { v10 }}$} & \multicolumn{2}{|c|}{$\begin{array}{l}\text { Safety } \\
\text { Evaluation }\end{array}$} & \multirow{2}{*}{$\frac{\text { Follow-up }}{\text { V16-22 }}$} \\
\hline & & $\mathrm{v} 1$ & v2 & & & & & & & & & $\mathrm{v} 10$ & $\mathrm{v} 11$ & \\
\hline Days/Months & & D-14 & $\mathrm{D}(-1)$ & D1 & D7 & D15 & D16 & $\mathrm{D} 22$ & D52 & D82 & D108 & M5 & M6 & M6-12 \\
\hline ICF & & $x$ & & & & & & & & & & & & \\
\hline I/E Criteria & $x$ & $x$ & & & & & & & & & & & & \\
\hline Infusion dates & & & & $x$ & & $x$ & & & & & & & & \\
\hline $\begin{array}{l}\text { Clinical } \\
\text { evaluation }\end{array}$ & $x$ & $x$ & $x$ & $x$ & $x$ & $x$ & & $x$ & $x$ & $x$ & $x$ & $x$ & $x$ & $x$ \\
\hline Clinical History & $x$ & $x$ & $x$ & $x$ & $x$ & $x$ & & $x$ & $x$ & $x$ & $x$ & $x$ & $x$ & $x$ \\
\hline Vital Signs & $x$ & $x$ & $x$ & $x$ & $x$ & $x$ & & $x$ & $x$ & $x$ & $x$ & $x$ & $x$ & $x$ \\
\hline $\begin{array}{l}\text { Physical } \\
\text { Examination }\end{array}$ & $x$ & $x$ & $x$ & $x$ & $x$ & $x$ & & $x$ & $x$ & $x$ & $x$ & $x$ & $x$ & $x$ \\
\hline ECG & $x$ & $x$ & $x$ & $x$ & $x$ & $x$ & & $x$ & $x$ & $x$ & $x$ & $x$ & $x$ & $x$ \\
\hline Chest X-ray & $x$ & & & & & & & & & & & & & \\
\hline QFT-G & $x$ & & & & & & & & & & & & & \\
\hline $\begin{array}{l}\text { General blood } \\
\text { work (CBC, } \\
\text { biochemical } \\
\text { profile, liver } \\
\text { essay) }\end{array}$ & & & $x$ & $x$ & $x$ & $x$ & & $x$ & $x$ & $x$ & $x$ & $x$ & $x$ & $x$ \\
\hline $\begin{array}{l}\text { Pregnancy tests } \\
\text { (urinalysis) }\end{array}$ & & & $x$ & $x$ & $x$ & $x$ & & $x$ & $x$ & $x$ & $x$ & $x$ & $x$ & $x$ \\
\hline $\begin{array}{l}\text { Cytokines and } \\
\text { inflammation } \\
\text { markers }\end{array}$ & & $x$ & & & $x$ & & & & & & & & $x$ & \\
\hline CC & & & $x$ & $x$ & $x$ & $x$ & & $x$ & $x$ & $x$ & $x$ & $x$ & $x$ & $x$ \\
\hline BNP & & & $x$ & $x$ & $x$ & $x$ & & $x$ & $x$ & $x$ & $x$ & $x$ & $x$ & $x$ \\
\hline Troponin I & & & & $x$ & $x$ & $x$ & & $x$ & $x$ & $x$ & $x$ & $x$ & $x$ & $x$ \\
\hline $\begin{array}{l}\text { Collagen } \\
\text { markers (PIIINP, } \\
\text { PINP, ICTP) }\end{array}$ & & & $x$ & & & & & & & & $x$ & & $x$ & \\
\hline Holter ECG 24 h & $x$ & & & $x$ & & $x$ & & & & & & & $x$ & $x$ \\
\hline NYHA & $x$ & $x$ & $x$ & $x$ & & & & & & & & & $x$ & $x$ \\
\hline Cardiac MRI & & & $x$ & & & & & & & & & & $x$ & $x$ \\
\hline $\begin{array}{l}\text { Minnesota Heart } \\
\text { Failure Survey }\end{array}$ & & $x$ & & & & & & & & & & & $x$ & $x$ \\
\hline
\end{tabular}

Figure 4 Study schedule. BNP, B-type natriuretic peptide; CC, creatinine clearance; ICF, informed consent form; I/E, inclusion and exclusion criteria; NYHA, New York Heart Association; QFT-G Quantiferon-G.

event of any AE. Constant monitoring must take place during these infusion, assessing vital signs every $15 \mathrm{~min}$ during the infusion of the study drug and up to an hour thereafter. All the while, the intravenous line will be maintained, enabling to proceed if any reaction occurs, as previously described by Specks et al. ${ }^{16}$ We will not administer rituximab as an intravenous push or bolus due to the risk of hypersensitive reactions that could ensue. We will obtain an ECG to assess any possible arrhythmogenic AE. Patients will later be discharged with a 24-hour Holter and return the next day for full clinical evaluation by the research team. There will be a 24 hours phone line and a mobile line available if patients require medical assistance.

As previously observed, an $\mathrm{H} 2$ histamine receptor antagonist (ranitidine orally, $150 \mathrm{mg}$ ), an antihistaminic (diphenhydramine orally, $50 \mathrm{mg}$ ) or an equivalent dose of a similar antihistamine, acetaminophen orally $650 \mathrm{mg}$ and methylprednisolone intravenous $100 \mathrm{mg}$ will be given orally $30 \mathrm{~min}$ before each infusion of rituximab. ${ }^{11}$ 
Premedication is known to attenuate infusion-related events. Because transient hypotension may occur during rituximab infusion, no antihypertensive medications will be administered at least 12 hours before the initiation of the infusion. The rituximab solution should be administered intravenously using infusion pumps at an initial rate of $50 \mathrm{mg} /$ hour. If hypersensitivity or infusion-related events do not occur, we shall escalate the infusion rate in $50 \mathrm{mg} /$ hour increments every $30 \mathrm{~min}$, to a maximum of $400 \mathrm{mg} /$ hour to fulfil the total dose of $1000 \mathrm{mg} .{ }^{15}$ If no adverse effects ensue, the total dose of $1000 \mathrm{mg}$ would be administered in 4 hours with $15 \mathrm{~min}$. If no reactions occur with the first infusion, subsequent rituximab infusions will be administered at an initial rate of $100 \mathrm{mg}$ / hour and then increased by $100 \mathrm{mg} /$ hour every $30 \mathrm{~min}$, to a maximum of $400 \mathrm{mg} /$ hour as tolerated..$^{15}$ If in this second infusion no adverse effects ensue, the total dose of $1000 \mathrm{mg}$ would be administered in 3 hours with $15 \mathrm{~min}$. Moreover, appropriate precautions should be used for the second infusion: during the second administration, the same safety strategy will be considered, even after subjects had not experienced any AE during first drug infusion.

\section{Common rituximab-associated not serious and serious adverse events}

Infusion reactions, ascribed to either allergic reactions or more seriously, anaphylaxis, were the most common AEs. Many reactions developed within 24hours, most frequently subsequently to the first infusion, which may be related to the release of cytokines or other chemical mediators, and rarely necessitate discontinuation of treatment. Clinically, these reactions include fevers, chills, rigours, nausea, dizziness, pruritus, urticaria/rash, angioedema, laryngeal oedema, sneezing, throat irritation/tightness, cough, hoarseness, bronchospasm, pulmonary infiltrates, hypoxia and acute respiratory insufficiency. ${ }^{15}$ A later and sometimes confusing $\mathrm{AE}$ is hypersensitivity pneumonitis presented as dyspnoea, hypoxaemia, eosinophilia and pulmonary infiltrates. ${ }^{18} 19$

When reactions are mild, the rate of infusion will be slowed or temporarily interrupted and resumed at half the previous rate after symptoms subsided. In the series of 465 patients treated for rheumatoid arthritis, serious infusion reactions decreased from $32 \%$ in the group receiving two $500 \mathrm{mg}$ infusions of rituximab and $37 \%$ in the group receiving two $1000 \mathrm{mg}$ infusions of rituximab to $19 \%$ and $29 \%$, respectively, after premedication with intravenous glucocorticoids. ${ }^{20}$ This indicates the importance and relevance of adequate premedication to minimise incidents and to increase patient safety.

\section{Possible increased risk of cardiac ischaemic events}

The proposed mechanism of rituximab-induced myocardial infarction could be the release of cytokines following the death of B-cells that leads to platelet activation, vasoconstriction and plaque rupture. ${ }^{21}$ The risk could be more in patients with pre-existing atherosclerosis and vulnerable plaques and advanced stage malignancy.
Adequate hydration before initiating rituximab (especially with first dose infusion) treatment might be a preventive strategy. Due to reported cases of angina, ACS and arrhythmias related to rituximab infusion, caution for its use is advised by the Food and Drug Administration in patients with a history of cardiovascular disease. ${ }^{17}$ In addition to ACS and arrhythmia, rituximab-induced cardiogenic shock and delayed reduction of LVEF, Takotsubo and non-ischaemic cardiomyopathies have also been described. ${ }^{18}$ The majority of the events have been described in rheumatoid arthritis. ${ }^{22}{ }^{23}$ Awareness of the cardiotoxicity profile of rituximab is important to minimise the risk of treatment-related morbidity/mortality. As described in detailed previously, in addition to ACS and cardiac arrhythmia, non-ischaemic cardiomyopathy may be associated with the infusion of rituximab, occurring within the first 48 hours. Symptomatology that could be attributed to ACS or HFrEF should be given special attention as a life-threatening event and must be ruled out. ${ }^{9}$

\section{Immunosuppression-related adverse effects}

Infections are an $\mathrm{AE}$ that must be detected early. Comorbidities that patients could have (ie, diabetes mellitus type 2) only increase the risk of complications such as sepsis, and sequelae. Six of the most severe infections are gastroenteritis, pyelonephritis, influenza, fever of unknown aetiology and de novo hepatitis B virus, colitis and pneumonia. ${ }^{24}$

\section{Management of adverse events}

In the case that despite all efforts, an AE occurs, efficient emergency care will be readily available at the infusion centre. Severe reactions typically occur during the first infusion with time to onset of 30-120 min. Rituximab-induced infusion reactions include: hypotension, angioedema, hypoxia, bronchospasm, pulmonary infiltrates, acute respiratory distress syndrome, myocardial infarction, ventricular fibrillation, cardiogenic shock, anaphylactic events or death. Hence, clinical staff will be aware of detecting these reactions. ${ }^{15}$ Intravenous antihistamine (chlorphenamine $10 \mathrm{mg}$ intravenous) and/ or glucocorticoids (hydrocortisone $100 \mathrm{mg}$ intravenous) will be used initially. In more severe cases (oedema of the face, dysphonia, stridor, respiratory failure or imminent collapse), epinephrine $(1 \mathrm{mg}=1 \mathrm{~mL}$ of epinephrine in aqueous solution $1 / 1000$ ) is $0.3-0.5 \mathrm{mg}$ in a single dose. This treatment will be repeated every $5-15$ min according to the severity and tolerance of the patient, plus continuous oxygen therapy, with monitoring of arterial $\mathrm{O}_{2}$ saturation, intravenous fluids and haemodynamic status monitoring, as in any anaphylaxis. If severe infusion reactions occur, the infusion of rituximab will be stopped. The infusion will be restarted after symptoms and laboratory abnormalities have resolved completely. If life-threatening anaphylactic or hypersensitivity reactions occur, participants will be removed from the treatment phase and not retreated with the study drug. Before infusions, a blood sample for complete blood count (CBC) will be 
collected and sent for analysis. If a participant's WBC count is $<3000 / \mathrm{mm}^{3}$, the infusion should be withheld. The CBC will be repeated weekly or as often as necessary for optimal medical care. If the participant's WBC count returns to $>3000 / \mathrm{mm}^{3}$, the next scheduled infusion of the rituximab will be administered. If the count does not rise above $3000 / \mathrm{mm}^{3}$ within 2 weeks, the participant will be excluded and will be treated according to best clinical judgement.

\section{Permitted dose adjustments and interruptions of study treatment}

The rituximab infusion should be interrupted if severe infusion reactions occur. In most cases of mild-to-moderate reactions, the infusion can be resumed at a $50 \%$ rate reduction (ie, from 100 to $50 \mathrm{mg}$ /hour) when all symptoms have improved entirely and are no longer deemed a threat to the participant well-being by the clinical staff. ${ }^{15}$ For example, some mild reactions (ie, flushing) may not be completely resolved despite reducing the infusion rate and administering additional acetaminophen and/or antihistamines. When the participant infusion reaction symptoms are deemed improved according to the clinical staff, the infusion rate can be resumed, as described.

All vital sign infusion reactions must be completely resolved before resuming the infusion rate as described. Treatment of mild-to-moderate symptoms with additional doses of antihistamines and acetaminophen is recommended..$^{15}$ Rigours can be managed with meperidine or morphine, depending on the site formulary. Additional treatment with bronchodilators or intravenous saline may be applied as clinically indicated. Epinephrine, antihistamines and glucocorticoids should be available for immediate use if a hypersensitivity reaction (ie, anaphylaxis) to rituximab occurs. The processes to carry out these procedures and a description of study instruments is described in detail and available in the safety, AEs and harms subsection along with the laboratory evaluation subsections of our data repository at Clinicaltrials.gov NCT03332888. Also, relevant concomitant care and interventions that are permitted or prohibited during the trial are also therein described.

\section{OUTCOMES}

\section{Primary outcomes}

Our primary outcome is the incidence of cardiovascular death, ACS, Takotsubo cardiomyopathy, worsening of LVEF, atrial and ventricular arrhythmias attributed to rituximab. Moreover, elevation of B-type natriuretic peptide $(\mathrm{BNP})>100 \mathrm{pg} / \mathrm{mL}$ or an increase of $1.5 \mathrm{x}$ in its basal expression and high-sensitivity cardiac troponin $\mathrm{I} \geq 2 \mathrm{ng} / \mathrm{L}$ values will be considered.

\section{Secondary outcomes}

As a secondary outcome, we will evaluate the concentrations of pretreatment and post-treatment biomarkers such as BNP, high-sensitivity cardiac troponin I, IL-1 $\beta$, interferon (IFN)- $\alpha$, IFN- $\gamma$, tumour necrosis factor (TNF)- $\alpha$, monocyte chemoattractant protein 1 (MCP-1), IL-6, IL-8, IL- 10, IL-12p70, IL-17A, IL-18, IL-23, IL-33, mononuclear cells, CD1d, CD19, CD5, CD20, IL-10, TNF- $\alpha$, PIIINP, PINP, ICTP. Finally, our exploratory objectives will be to evaluate the regression of the local cardiovascular, inflammatory response and prevent the progression of fibrosis, which would preserve the dimensions of the left ventricle, EF and the same degree of fibrosis. After an acute myocardial infarction, in heart failure and even in subjects without overt cardiovascular disease, the basal dimensions of the left ventricle and EF are significant predictors of survival. Therefore, these objectives will be measured to estimate the beneficial effects, similar to ACE inhibitors and beta-blockers that in the treatment of heart failure seem to possess the ability to inhibit or reverse cardiac remodelling. ${ }^{25}$ The images obtained by MRI with late enhancement of gadolinium (LGE) will be used to determine and describe the cardiac remodelling and, in doing so, to evaluate if the exploratory objectives of this work are met. These objectives, such as EF and myocardial fibrosis, will be evaluated using MRI. The method used on MRI to quantify myocardial fibrosis will be the clinically validated LGE. ${ }^{26}$ LGE measures if the retention of gadolinium contrast agent is prolonged compared with the healthy myocardium, resulting in the increased signal intensity of this region on a T1-weighted MRI image, by adding an inversion pulse with a well-chosen inversion time to null the signal of the healthy myocardium. Replacement fibrosis, (ie, due to ischaemic heart disease) can be distinguished as it is well-delineated on LGE images. Inflammation can and will also be detected; here contrast agents that infiltrate immunocompetent cells have been used, allowing these cells to be tracked and, as a consequence, effectively delimiting inflammatory regions. ${ }^{27}$ Also, the Minnesota Living with Heart Failure Questionnaire will be considered to assess the quality of life.

\section{Biomarkers}

During the study, the biomarkers will be categorised according to their purpose and relevance in ventricular dysfunction, fibrosis, micronecrosis and inflammation. For ventricular dysfunction, BNP will be considered for acute cellular damage, high-sensitivity cardiac troponin I will evaluate the presence of acute cellular damage/micronecrosis. The high-sensitivity cardiac troponin I assay is based on a enzyme linked fluorescent assay. Cardiac troponin I in the sample will bind to the specific antitroponin I antibodies, which are coated on the solid phase receptade, causing agglutination. The degree of the turbidity caused by agglutination can be measured optically and is proportional to the amount of troponin I in the sample. The reference range is $<2 \mathrm{ng} / \mathrm{L}$. Regarding BNP measurements, the methodology applied was immunochemiluminometric assay, where we consider a value $>100 \mathrm{pg} / \mathrm{mL}$ as elevated. Both will be measured at each visit for 
patient safety. A specific altruistic sample will be taken to analyse these biomarkers. Furthermore, in order to quantify the inflammatory behaviour and the effect of AMF-CD20, IL-1 $\beta$, IFN- $\alpha$, IFN- $\gamma$, TNF- $\alpha$, MCP-1 will be evaluated, IL-6, IL-8, IL-10, IL-12p70, IL-17A, IL-18, IL-23, IL-33 as well as mononuclear cells, CD1D, CD19, CD5 and CD20. Finally, fibrosis will be considered as collagen markers N-Terminal Propeptide of Type III Collagen (PIIINP), N-Terminal Propeptide of Type I Collagen (PINP), C-Terminal Telopeptide Type I Collagen (ICTP). The panel of biomarkers of inflammation, mononuclear cells and fibrosis will be taken at the beginning, and at the end of the study.

\section{Concomitant treatment}

Every single concomitant medicine is taken by or controlled to subjects in the last 30 days before enrolment will be considered. All medications will be coded using the WHO drug dictionary. The number and percentage of subjects accepted earlier, and concomitants medications or treatments will be presented overall by prescription class.

\section{Statistical design and analysis}

The continuous variables will be presented as median and IQR, and the categorical variables as frequencies and percentages, respectively. We will conduct statistical comparisons between the initial data from the baseline visit with data from the third and sixth months, respectively. The analysis of the primary end point will be descriptive, considering the incidence of related cardiovascular drug $\mathrm{AE}$ and it will be performed according to the on-treatment method. Comparisons and associations between demographic variables, laboratory results, measures of cardiovascular function and biomarkers over the prespecified visits will be performed using non-parametric tests (Wilcoxon signed rank test), depending on the distribution of variables. The analysis will be adjusted with regression methods for age, sex, body mass index and NYHA functional class. These results will be considered as exploratory. Statistical significance will be assumed with a one-sided $p$ value $<0.05$.

\section{Ethics and equipoise}

The trial was approved by IRB (registry number: CONBIOETICA-19-CEI-011-20161017 and COFEPRIS 17 CI 19039003 ) and is currently in the prerecruitment stage. This trial is executed in a group of patients, that due to their cardiovascular state, and functional class, their condition proves debilitating and incapacitating. Moreover, these patients, unfortunately, have a reserved prognosis, with an elevated 5-year mortality rate, and no known therapy that could lead to improvement. Hence, this trial searches to establish the safety of an alternative strategy in ameliorating prognosis, detaining heart failure progression through processes described anteriorly and a possibly better quality of life.

\section{Access to data and confidentiality}

Regarding the informed consent form data, the patient or the patient's legally authorised representative will receive a copy of each signed consent form. All signed and dated forms of consent will remain in the study file of each patient or centre file and will be available for study monitors to verify at any time. The data obtained from collecting lab samples, cardiovascular studies (ECG, echocardiography, 24-hour Holter), chest X-rays and altruistic samples will be stored in individual patient files. These files will be available to the principal investigator both in physical and electronic form. He will disclose the necessary information to subinvestigators when it is deemed necessary for the patient well-being and cardiovascular integrity. However, personal information of patients will be blinded to investigators, to preserve the confidentiality of patients. The patient's medical records from the trial such as baseline laboratory values, images and questionnaires will be used and entered into a database. Range checks will be done for all the data values, and a clinical description will be made for all image studies, which will in turn also be inserted into the database. We reiterate that patient's medical files will be managed professionally, releasing information only if deemed necessary by the principal investigator, at the benefit and the discretion of the patient while maintaining patient confidentiality. Any personal information of the patients will be blinded to the investigators.

\section{Ancillary and post-trial care}

If necessary, ancillary care will be covered at the Zambrano Hellion Hospital. In case of any AE, related to the study drug or not, patients who participate in this study will be able to rely on the Zambrano Hellion Hospital for prompt admission and care. They will be promptly admitted to medical care in the event of any medical emergency or complication, and the institutional account 0010RPD003 will cover all the medical expenses.

\section{Protocol amendments}

Any amendment that this protocol could be submitted to will be reported and sent to the Ethics and Research Committees of the Tecnologico de Monterrey for further evaluation, who according to National guidelines and policy, will assess these changes.

\section{ICF PROCEDURES}

All investigators are required to carry out an exhaustive expounding of each procedure to participants and acquire the patient's signature in the presence of a witness. The ICF form will contain different sections, each hosting its designated procedure, consent provisions for collection and use of participant data and biological specimens in ancillary studies. The investigator will explain to each patient the objectives, methods and possible risks associated with the procedure involved. Patients must be informed that they have the right to withdraw their 
consent at any time and for any reason. A separate signature will be required to document a patient's agreement to participate in collection and use of participant data and biological specimens in ancillary studies. The patient or the legally authorised representative will sign and date forms of consent before their participation in the study. Patients required to co-sign the most recent version of consent forms during their participation in the study. In the case of modified or updated consent forms, the case history or clinical records of each patient must document this change to be allowed to continue participating in the study. The patient or the patient's legally authorised representative will receive a copy of each signed consent form. All signed and dated forms of consent will remain in the study file of each patient or centre file and will be available for study monitors to verify at any time. These ICF forms will be reviewed, if/when changes are made to a research procedure or with the genesis of newly available information that could affect the patient's willingness to participate.

\section{DISCUSSION}

Heart failure syndromes are affecting about 26 million people worldwide, and half of the population has a 5-year mortality rate. ${ }^{28}$ Its prevalence from 2009 to 2012 in the USA was 5.7 million people, from 2011 to 2014 there was an increase to 6.5 million Americans. It is expected that by 2030 the increase will be $46 \%$, almost 8 million Americans. ${ }^{28}{ }^{29}$ Additionally, heart failure syndromes are the fourth leading cause of adult hospitalisations and the most frequent cause of hospitalisation in patients older than 65 years. ${ }^{28}{ }^{29}$ The 1-year mortality in patients with heart failure syndromes is over $30 \%$ in NYHA functional class III and IV and $>60 \%$ in class IV. ${ }^{30}$ Mortality is related to ventricular arrhythmias, recurrent myocardial infarction, electrolyte disturbances and the use of psychotropic and antiarrhythmic drugs. Atrial fibrillation, a common complication, increases stroke and thromboembolic complications. ${ }^{31}$

Among heart failure syndromes, HFrEF is the final common pathway of multifactorial triggers (ie, toxic, infectious, genetic). The pathophysiological mechanisms are characterised by cardiomyocyte damage, endothelial dysfunction leading to microvascular damage, inadequate ventricular remodelling, ventricular dysfunction as well as the activation of the vasopressin and renin-angiotensin-aldosterone system. ${ }^{2}{ }^{32}$ The clinical presentation includes a progressive clinical deterioration from a subclinical stage, characterised by initial clinical stability, in the absence of symptoms, to a clinical stage with frequent exacerbations, marked deterioration of functional status and quality of life and finally, premature mortality due to left ventricular dysfunction and sudden arrhythmogenic death.

Although available therapeutic approaches based on neurohormonal inhibition ${ }^{32}$ have shown a significant decrease in HFrEF-related mortality, currently, despite treatment with evidence-based chronic HFrEF therapy during hospitalisation, postdischarge AEs, rehospitalisation and mortality event rates remain high. On the other hand, clinical and experimental data support the additional critical role that chronic inflammation plays in HFrEF ${ }^{33-35}$ An increase in pro-inflammatory cytokines has been identified in the later stages of HFrEF, which can be considered as evidence of innate and acquired immune system activity. Previously, we found elevated levels of TNF- $\alpha$ in patients with advanced congestive heart failure. ${ }^{33}$ We presume that along with other pro-inflammatory cytokines, it could lead to left ventricular dysfunction and cardiomyopathy as seen in animal models. ${ }^{33} 34$

Recently, we demonstrated myocardial-associated antibody profiles in patients with severe HFrEF, for the first time. ${ }^{1}$ In this study, we obtained cardiac biopsies from two groups: 100 from patients with end-stage heart failure and 40 from healthy donor patients, where we confirmed the presence of increased levels of IgG and its antigen in the hearts of the end-stage heart failure group. We localised IgG in $71 \%$ of these patients, $48 \%$ of those being positive for the subtype IgG3. The proportion of patients with ischaemic heart disease that were positive for IgG was $65 \%$, and among patients with non-ischaemic aetiologies, it was $76 \%{ }^{1}$. The complement system was activated in $31 \%$ of all patients. The presence of IgG and C3 was directly correlated with the length and progressive state of disease $(\mathrm{r}=0.451, \mathrm{p}=0.006)$. We also identified the ATP synthase b-subunit as a prominent anticardiac antibody and antibody deposition which was largely encountered on the sarcolemma, indicating that the antibodies were more likely anticardiac antibodies. This finding was strengthened by the demonstration of co-localised Ig3 and C3c deposits in a similar distribution on the sarcolemma. Importantly, immune activation was demonstrated as a late event in the course of heart failure as indicated by a higher proportion of IgG3 and C3c deposition in patients with a more prolonged duration of illness. The formation of anticardiac antibodies does not appear to be a phenomenon exclusive to patients with non-ischaemic aetiology since we found similar proportions of positive cardiac samples among ischaemic and non-ischaemic patients. These findings provide important and new information about the role of the humoral immune responses in the progression of heart failure. ${ }^{1}$ In a significant proportion of other studied tissues, complement components were activated on their encounter as evidence of local inflammatory activity. ${ }^{136}$ This evidence suggests that in the genesis of HFrEF, there is a B-lymphocyte response to the release of cardiac proteins. The role of B-cells as potential contributors to cardiac injury depends on whether B-cells or their products respond to the neoexpression of altered cardiac proteins, exposed to the immune system. ${ }^{36} \mathrm{We}$ recently encountered examples of B-cells as the primary mediators of lesions that can be found in patients with dilated cardiomyopathy in whom circulating antibodies have been demonstrated to possess activity against the $\beta$-receptor, in which the elimination of these antibodies significantly improves the course of the disease. ${ }^{236}$ 
Our observations provide us with a greater knowledge of the immunological processes involved in HFrEF, which leads us to consider this syndrome as a neurohumoral inflammatory pathology. ${ }^{4}$ Additionally, we found that the pharmacological depletion of B-lymphocytes with a monoclonal antibody directed against the CD20 B-cell marker reduced the expression of heart failure in mice, showing decreased fibrosis, ventricular dilatation, resulting in an improvement in the ejection fraction. ${ }^{5}$ Importantly, rituximab, a humanised monoclonal antibody anti-CD20 (HMA-CD20) has shown efficacy and safety in haematological malignancies and autoimmune diseases, by depleting overall B-cell count. Besides, we observed a regression of ventricular dysfunction when these circulating antibodies were neutralised in postheart-transplant patients complicated by severe heart failure due to transplant rejection. ${ }^{37-41}$ These observations suggest that the inflammatory phenomenon plays a central role in the genesis and progression of heart failure and that it may also be reversible. Based on this evidence, it could be considered that HMA-CD20 might aid in the depletion of activated B-cells in patients with severe HFrEF, and therefore possibly decrease myocardial damage progression and maintain ventricular function. Due to the fragile nature of this population, and absent evidence of prior use of HMA-CD20 in this disease, the primary target of our study will be to determine the safety of HMA-CD20 use in patients with severe HFrEF that have an inadequate response to conventional treatment.

Contributors LS-T provided substantial contributions to the conception and design of the project. Also, he was involved in the planning, data resourcing and overall elaboration of the protocol and the Informed Consent Form. Additionally, to his aforementioned contributions, he played an important role in the consensus and ultimate approval of the version to be published and agreed to be accountable for all aspects of the work. CJ-S contributed to the conception and study design. He collaborated in drafting and revising the work for critically for important intellectual content. Additionally, to his contributions above, he played an important role in the ultimate approval of the version to be published and agreed to be accountable for all aspects of the work. DR had contributions to the conception and design of the work while playing a defining role in the methodology for the eventual acquisition, analysis and interpretation of data for the work. He also aided in critically revising the work, ensuring the homogeny and integrity of the content. Additionally, to his contributions above, he played an important role in the ultimate approval of the version to be published and agreed to be accountable for all aspects of the work. JP contributed to the study design, aiding in critically reviewing the work, ensuring the integrity of the content. Additionally, to his contributions above, he played an important role in the ultimate approval of the version to be published and agreed to be accountable for all aspects of the work. $\mathrm{CO}$ - L contributed to the study design, aiding in critically reviewing the work, ensuring the integrity of the content. Additionally, to her aforementioned contributions, she played an important role in the ultimate approval of the version to be published and agreed to be accountable for all aspects of the work. GG-R was responsible for the conception and the study design. He collaborated in drafting and revising the work critically for important intellectual content. Additionally, to his contributions above, he also approved the final version to be published and agreed to be accountable for all aspects of the work. GT-A substantially contributed to the conception of idea and design of the study. Drafting the work and revising it critically for essential intellectual content. He has agreed to be held accountable for all the aspects of the work and ensures that questions related to the accuracy or integrity of any part of the work are appropriately investigated and resolved.

Funding Because this is an investigator-initiated study, funding will be provided internally, all expenses will be covered by the Centro de Investigación Biomédica del Hospital Zambrano Hellion (Biomedical Investigation C enter of the Zambrano
Hellion Hospital), with the institutional account 0010RPD003. This research received no specific grant from any funding agency in public, commercial or not-for-profit sectors.

\section{Competing interests None declared.}

Patient consent for publication Not required.

Ethics approval The study was approved by the Comite de Etica en Investigacion de la Escuela de Medicina del Instituto Tecnologico y de Estudios Superiores de Monterrey and the Comite de Investigacion de la Escuela de Medicina del Instituto Tecnologico y de Estudios Superiores de Monterrey.

Provenance and peer review Not commissioned; externally peer reviewed.

Open access This is an open access article distributed in accordance with the Creative Commons Attribution Non Commercial (CC BY-NC 4.0) license, which permits others to distribute, remix, adapt, build upon this work non-commercially, and license their derivative works on different terms, provided the original work is properly cited, appropriate credit is given, any changes made indicated, and the use is non-commercial. See: http://creativecommons.org/licenses/by-nc/4.0/.

\section{REFERENCES}

1. Youker KA, Assad-Kottner C, Cordero-Reyes AM, et al. High proportion of patients with end-stage heart failure regardless of aetiology demonstrates anti-cardiac antibody deposition in failing myocardium: humoral activation, a potential contributor of disease progression. Eur Heart J 2014;35:1061-8.

2. Sánchez-Trujillo L, Vázquez-Garza E, Castillo EC, et al. Role of Adaptive Immunity in the Development and Progression of Heart Failure: New Evidence. Arch Med Res 2017;48:1-11.

3. Kaya Z, Leib C, Katus HA. Autoantibodies in heart failure and cardiac dysfunction. Circ Res 2012;110:145-58.

4. Ludwig RJ, Vanhoorelbeke K, Leypoldt F, et al. Mechanisms of Autoantibody-Induced Pathology. Front Immunol 2017;8.

5. Cordero-Reyes AM, Youker KA, Trevino AR, et al. Full Expression of Cardiomyopathy Is Partly Dependent on B-Cells: A Pathway That Involves Cytokine Activation, Immunoglobulin Deposition, and Activation of Apoptosis. J Am Heart Assoc 2016;5:e002484.

6. Aggarwal A, Pyle J, Hamilton J, et al. Low-dose rituximab therapy for antibody-mediated rejection in a highly sensitized heart-transplant recipient. Tex Heart Inst J 2012;39:901-5.

7. Ridker PM, Everett BM, Thuren T, et al. Antiinflammatory Therapy with Canakinumab for Atherosclerotic Disease. N Engl J Med 2017;377:1119-31.

8. Roy A, Khanna N, Senguttuvan NB. Rituximab-vincristine chemotherapy-induced acute anterior wall myocardial infarction with cardiogenic shock. Tex Heart Inst J 2014;41:80-2.

9. Cheungpasitporn W, Kopecky SL, Specks U, et al. Non-ischemic cardiomyopathy after rituximab treatment for membranous nephropathy. J Renal Inj Prev 2017;6:18-25.

10. Umscheid CA, Margolis DJ, Grossman CE. Key concepts of clinical trials: a narrative review. Postgrad Med 2011;123:194-204.

11. Rubbert-Roth A, Tak PP, Zerbini C, et al. Efficacy and safety of various repeat treatment dosing regimens of rituximab in patients with active rheumatoid arthritis: results of a Phase III randomized study (MIRROR). Rheumatology 2010;49:1683-93.

12. Theuer CP, Leigh BR, Multani PS, et al. Radioimmunotherapy of nonHodgkin's lymphoma: Clinical development of the Zevalin regimen. In: Biotechnology Annual Review. Elsevier, 2004:265-95.

13. Oncology. In: Nuclear Medicine. Elsevier, 2014:265.

14. Havrdova E, Horakova D, Kovarova I. Alemtuzumab in the treatment of multiple sclerosis: key clinical trial results and considerations for use. Ther Adv Neurol Disord 2015;8:31-45.

15. Kasi PM, Tawbi HA, Oddis CV, et al. Clinical review: Serious adverse events associated with the use of rituximab - a critical care perspective. Crit Care 2012;16:231.

16. Specks U, Merkel PA, Seo P, et al. Efficacy of remissioninduction regimens for ANCA-associated vasculitis. N Engl J Med 2013;369:417-27.

17. Zhang Y, Vermeulen LC, Kolesar JM. Stability of stock and diluted rituximab. Am J Health Syst Pharm 2013;70:436-8.

18. Mohrbacher A. B cell non-Hodgkin's lymphoma: rituximab safety experience. Arthritis Res Ther 2005;7 Suppl 3:S19-25.

19. Omri HE, Taha RY, Gamil A, et al. Efficacy and safety of rituximab for refractory and relapsing thrombotic thrombocytopenic purpura: a cohort of 10 cases. Clin Med Insights Blood Disord 2015;8:CMBD. S25326. 
20. Dotan E, Aggarwal C, Smith MR. Impact of Rituximab (Rituxan) on the Treatment of B-Cell Non-Hodgkin's Lymphoma. P T 2010;35:148-57.

21. Aronson JK. Meyler's side effects of cardiovascular drugs. 1st ed. Amsterdam: Boston, Mass: Elsevier, 2009. http://www.clinicalkey. com/dura/browse/bookChapter/3-s2.0-C20090017875. (accessed 10 Nov 2017).

22. Ng KH, Dearden C, Gruber P. Rituximab-induced Takotsubo syndrome: more cardiotoxic than it appears? BMJ Case Rep 2015;2015.

23. Agca R, Heslinga SC, Rollefstad S, et al. EULAR recommendations for cardiovascular disease risk management in patients with rheumatoid arthritis and other forms of inflammatory joint disorders: 2015/2016 update. Ann Rheum Dis 2017;76.

24. Randall KL. Rituximab in autoimmune diseases. Aust Prescr;39:131-4.

25. Ponikowski P, Voors AA, Anker SD, et al. ESC Scientific Document Group. 2016 ESC Guidelines for the diagnosis and treatment of acute and chronic heart failure: The Task Force for the diagnosis and treatment of acute and chronic heart failure of the European Society of Cardiology (ESC)Developed with the special contribution of the Heart Failure Association (HFA) of the ESC. Eur Heart $J$ 2016;37:2129-200.

26. Haaf P, Garg P, Messroghli DR, et al. Cardiac T1 Mapping and Extracellular Volume (ECV) in clinical practice: a comprehensive review. J Cardiovasc Magn Reson 2016;18:89.

27. van Oorschot JW, Gho JM, van Hout GP, et al. Endogenous contrast MRI of cardiac fibrosis: beyond late gadolinium enhancement. $J$ Magn Reson Imaging 2015;41:1181-9.

28. Savarese G, Lund LH. Global public health burden of heart failure. Card Fail Rev 2017;03:7.

29. Vinson JM, Rich MW, Sperry JC, et al. Early readmission of elderly patients with congestive heart failure. J Am Geriatr Soc 1990;38:1290-5.

30. Lipicky RJ, Packer M. Role of surrogate end points in the evaluation of drugs for heart failure. J Am Coll Cardiol 1993;22:A179-A184.
31. Verma A, Kalman JM, Callans DJ. Treatment of Patients With Atrial Fibrillation and Heart Failure With Reduced Ejection Fraction. Circulation 2017;135:1547-63.

32. Greenberg B, Quinones MA, Koilpillai C, et al. Effects of long-term enalapril therapy on cardiac structure and function in patients with left ventricular dysfunction. Results of the SOLVD echocardiography substudy. Circulation 1995;91:2573-81.

33. Torre-Amione G, Kapadia S, Lee J, et al. Tumor necrosis factoralpha and tumor necrosis factor receptors in the failing human heart. Circulation 1996;93:704-11.

34. Feiner EC, Chung P, Jasmin JF, et al. Left ventricular dysfunction in murine models of heart failure and in failing human heart is associated with a selective decrease in the expression of caveolin-3. J Card Fail 2011;17:253-63.

35. Flores-Arredondo JH, García-Rivas G, Torre-Amione G. Immune modulation in heart failure: past challenges and future hopes. Curr Heart Fail Rep 2011;8:28-37.

36. Epelman S, Liu PP, Mann DL. Role of innate and adaptive immune mechanisms in cardiac injury and repair. Nat Rev Immunol 2015;15:117-29.

37. Imamura T, Kinugawa K, Kato N, et al. Successful treatment of hemodynamic compromise caused by antibody-mediated and cellular rejection in a recipient 12 years after heart transplantation. Int Heart J 2013;54:328-31.

38. Ravichandran AK, Schilling JD, Novak E, et al. Rituximab is associated with improved survival in cardiac allograft patients with antibody-mediated rejection: a single center review. Clin Transplant 2013;27:961-7.

39. Toscano G, Tartaro P, Fedrigo M, et al. Rituximab in recurrent idiopathic giant cell myocarditis after heart transplantation: a potential therapeutic approach. Transpl Int 2014;27:e38-e42.

40. Asante-Korang A, Jacobs JP, Ringewald J, et al. Management of children undergoing cardiac transplantation with high Panel Reactive Antibodies. Cardiol Young 2011;21 Suppl 2:124-32.

41. Patel JK, Kobashigawa JA. Improving survival during heart transplantation: diagnosis of antibody-mediated rejection and techniques for the prevention of graft injury. Future Cardiol 2012;8:623-35. 